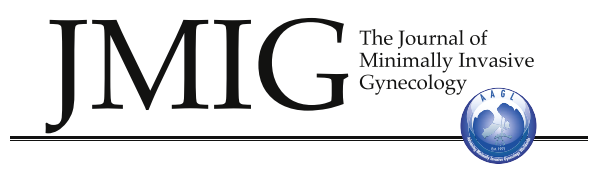

Original Article

\title{
One-Year Follow-Up Results of a Multicenter, Single-Arm, Objective Performance Criteria-Controlled International Clinical Study of the Safety and Efficacy of the Minerva Endometrial Ablation System
}

\author{
Philippe Laberge, MD, FRCSC, ACGE*, Jose Garza-Leal, MD, Claude Fortin, MD, FRCSC, \\ Robert Sabbah, MD, FRCSC, Tamas Fulop, MD, Norbert Pásztor, MD, and György Bacsko, MD \\ From the Department of Obstetrics and Gynecology, Laval University, Quebec City, QC, Canada (Dr. Laberge), Department of Obstetrics and Gynecology, \\ Universidad Autonoma de Nuevo Leon, Monterrey, NL, Mexico (Dr. Garza-Leal), Department of Obstetrics and Gynecology, Hôpital de LaSalle, LaSalle, \\ QC, Canada (Dr. Fortin), Department of Obstetrics and Gynecology, Hôpital du Sacre-Coeur de Montreal, Montreal, QC, Canada (Dr. Sabbah), \\ Department of Obstetrics and Gynecology, Szent Imre Kórház, Budapest, Hungary (Dr. Fulop), Department of Obstetrics and Gynecology, University of \\ Szeged, Szent-Györgyi Albert Medical Center, Szeged, Hungary (Dr. Pásztor), and Department of Obstetrics and Gynecology, Kenezy Korház, Debrecen, \\ Hungary (Dr. Bacsko).
}

ABSTRACT Study Objective: To assess the safety and effectiveness of the Minerva endometrial ablation system for treating excessive uterine bleeding in premenopausal women.

Design: Multicenter, single-arm, objective performance criteria (OPC)-controlled international study (Canadian Task Force classification II-1).

Setting: Seven academic medical centers.

Patients: 105 premenopausal women symptomatic for menorrhagia secondary to dysfunctional uterine bleeding.

Intervention: Patients were treated using the Minerva endometrial ablation system.

Measurements and Main Results: Study success, based on a pictorial blood loss assessment chart (PBLAC) score $\leq 75$, was observed in $96.2 \%$ of the patients at 1 year posttreatment. Some $69.5 \%$ of the patients experienced amenorrhea (PBLAC score 0 ). The mean duration of the procedure was 3.9 minutes. General anesthesia was used in $9 \%$ of cases, with the balance being performed under local and/or intravenous or spinal anesthesia regimens. No intraoperative adverse events and/or complications were reported. No patient required hysterectomy or any additional medical and/or surgical interventions to control bleeding during 1 year of follow-up. Efficacy (success) results were compared between the Minerva system and the OPC, which served as a statistical control. The OPC comprised the US Food and Drug Administration's (FDA) reported success rates of all FDA-approved endometrial ablation systems. The Minerva system had a statistically significantly superior success rate compared with the OPC control.

Conclusion: The Minerva system was found to be safe and effective for treating patients suffering from menorrhagia. The procedure is quick and effective, does not require endometrial pretreatment, and precludes the need for additional surgical interventions to manage menorrhagia. Journal of Minimally Invasive Gynecology (2015) $\mathbf{\square}, \mathbf{\square} \mathbf{\square}$ (c) 2015 AAGL. All rights reserved.

Keywords: $\quad$ Dysfunctional uterine bleeding; Endometrial ablation; Excessive menstrual bleeding; Hysteroscopy; Menorrhagia; Minerva Surgical; Objective performance criteria; Pictorial blood loss assessment chart

DISCUSS You can discuss this article with its authors and with other AAGL members at http:// www.AAGL.org/jmig-22-6-JMIG-D-15-00207.

Corresponding author: Philippe Laberge, MD, FRCSC, ACGE, Department of Obstetrics and Gynecology, Laval University, 2705 Blvd Laurier, S-768, Québec, QC G1V 4G2, Canada.

E-mail: philippe.laberge@fmed.ulaval.ca

Submitted April 3, 2015. Accepted for publication June 3, 2015.

Available at www.sciencedirect.com and www.jmig.org

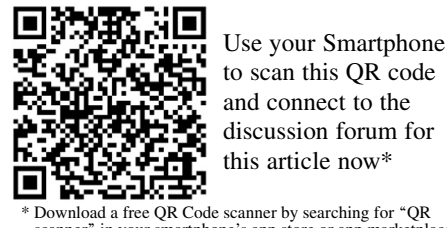

scanner" in your smartphone's app store or app marketplace.
Since the first reports by Goldrath et al [1], followed by DeCherney et al [2], Vancaillie [3], and others, endometrial ablation is now used worldwide as a safe and less invasive alternative to hysterectomy for the management of menorrhagia. Despite its early promise, however, the initial adoption of this technique was slowed by the general lack of 
advanced hysteroscopic and resectoscopic skills, coupled with concerns about the use of nonelectrolyte solutions for uterine distention [4,5]. Fueled by the unmet need, a generation of nonhysteroscopic devices was introduced to ablate the endometrium using safer and less skill-intensive methods. Following US Food and Drug Administration (FDA) approval of the first such device, the ThermaChoice UBT in 1997, others, including Her Option (cryosurgery), HydrothermAblator (hot saline), NovaSure (bipolar radiofrequency $[R F]$ ), and MEA (microwave energy), were sequentially approved. Using a prospective randomized controlled trial (RCT) design for each pivotal trial, the effectiveness and safety of each method was shown to be noninferior to that of rollerball endometrial ablation [6-15].

Despite the fact that endometrial ablation has been established as a safe and conservative alternative to hysterectomy and its use continues to expand [16], the impact of this less invasive procedure on the overall rate of hysterectomy has not been significant to date. Recent US data indicate that the hysterectomy rate has remained relatively constant at 600000 cases/year [17]. There are also concerns about the interval surgical interventions, such as reablation and/or hysterectomy, performed for recurrent menorrhagia and/or new pelvic pain [18-20].

The Minerva endometrial ablation system (Minerva Surgical, Redwood City, CA) was developed with the goal of exceeding established outcomes by using a novel technology. The Minerva system has been evaluated in a number of extirpated and perihysterectomy studies aimed at assessing the system's safety and efficacy. As such, the primary objective of the present study was to evaluate the safety and effectiveness of the Minerva endometrial ablation system, as evidenced by a reduction of bleeding to a normal menstrual blood loss level (defined as a score of $\leq 75$ on a pictorial blood loss assessment chart [PBLAC]) at 12 months posttreatment and the absence of any adverse events. Secondary objectives included amenorrhea rate, treatment parameters (procedure time, anesthesia type), patient satisfaction, and responses to a validated quality of life (QoL) questionnaire.

\section{Materials and Methods}

\section{General}

As part of the premarket approval submission to the FDA, the results of this study were used to assess the safety and efficacy of the Minerva endometrial ablation system. This investigation was a prospective, single-arm, multicenter, objective performance criteria (OPC)-controlled international study. The study was conducted at 7 academic centers in Canada, Mexico, and Hungary in accordance with the International Conference on Harmonisation's good clinical practices (GCP), including recommendations guiding physicians in biomedical research involving human subjects adopted by the 18th World Medical Assembly (1964 and later revisions) and standard ISO14155. In addition, the study design and execution complied with regulatory requirements developed and adopted by the FDA. The study protocol, informed consent document, case report forms, and other relevant documentation were all reviewed and approved by pertinent institutional review boards and country-specific regulatory bodies. Adherence to the GCP guidelines was monitored by ICRC, Inc (Encinitas, CA), an independent clinical research organization. To ensure statistical integrity and to avoid site-to-site crosscontamination, the data collection and management were conducted by a separate, independent clinical research organization (Medpace, Inc, Cincinnati, OH). Data entry and overall management were performed using Clindex clinical trial software (Fortress Medical Systems, Hopkins, MN).

Study progress was also critically monitored by 2 independent entities composed of experts in the field of gynecology and endometrial ablation. A Data Safety Monitoring Board (DSMB) was tasked to oversee the overall progress and safety of the study. A Clinical Events Committee (CEC) assessed and ultimately adjudicated all observed adverse events with respect to severity and relationship to the device and/or the procedure. Both the DSMB and the CEC had the authority to recommend study termination in the event that patient safety was compromised and/or the efficacy data did not support or justify future recruitment of subjects.

\section{Clinical Protocol}

The primary objective of the present study was to evaluate the safety and effectiveness of the Minerva endometrial ablation system, as evidenced by reduction in bleeding to a normal menstrual blood loss level (defined as a PBLAC score $\leq 75)$ at 12 months posttreatment and the absence of adverse events. Secondary objectives included amenorrhea rate, treatment parameters (procedure time, anesthesia type), patient satisfaction, and responses to validated QoL and menstrual impact questionnaires.

\section{Blood Loss Assessment}

Menstrual bleeding volume was assessed using the PBLAC scoring system described by Higham et al [21]. Using the Higham methodology, a PBLAC score of 100 has been shown to have a sensitivity of $86 \%$ and a specificity of $89 \%$ for the diagnosis of menorrhagia ( $>80 \mathrm{~mL}$ menstrual blood loss per cycle). Before study initiation, a separate sanitary product validation study was conducted to ensure accurate correlation between the absorption of each sanitary product and the Higham scoring method. Study subjects were instructed to use only validated sanitary products, which were provided free of charge. Bleeding levels were assessed preoperatively, and all candidates had to satisfy a minimum PBLAC score of 150 per cycle to qualify for study participation. 
Postoperatively, menstrual bleeding score was assessed monthly from month 2 through month 12 . Patients reporting amenorrhea were instructed to document the total absence of bleeding on each monthly diary.

\section{Inclusion/Exclusion Criteria}

Subjects eligible for study participation were required to be premenopausal (follicle-stimulating hormone level $<40$ $\mathrm{mIU} / \mathrm{mL}$ ), between 25 and 50 years of age, have no desire for fertility, and a documented PBLAC score confirming menorrhagia without a definable cause. Uterine sounding length was limited to $10 \mathrm{~cm}$ and cavity width to no smaller than $2.5 \mathrm{~cm}$. Subjects were not permitted to use any form of hormonal birth control during the follow-up period, to eliminate the possibility that posttreatment bleeding reduction was induced by and/or attributed to the suppressive action of hormonal contraceptives. Subjects were required to demonstrate literacy and the ability to accurately use menstrual diaries.

Subjects were excluded if they presented with evidence of pelvic inflammatory disease, active/acute endometritis, sexually transmitted disease, bacteremia, sepsis, other active local and/or systemic infection, untreated/unevaluated cervical dysplasia (except CIN I), endometrial hyperplasia, or known or suspected abdominal or pelvic cancer. Subjects with suspected or known coagulopathies, receiving anticoagulation therapy, or diagnosed with a congenital malformation of the uterus, fibroid(s) distorting the uterine cavity, or large endometrial polyp(s), and those less than 6 weeks postpartum were excluded from study participation.

\section{Statistics and the OPC Control}

This investigation was designed as a single-arm study comparing the effectiveness of the Minerva endometrial ablation system with that of the OPC control recommended by the FDA. The analysis of success rates for the 5 previously approved endometrial ablation devices was performed and provided by the FDA. Based on these data (Her Option, 67.4\%; HydroThermablator, 68.4\%; Microwave Endometrial Ablation/MEA, 87\%; NovaSure, 77.7\%; ThermaChoice, $75.4 \%$ ), the calculated least squares mean success rate for the OPC was 75.6\%, with lower and upper 95\% confidence bounds of $65.6 \%$ and $83.5 \%$, respectively (Fig. 1). The study sample size to obtain at least $80 \%$ power, thereby detecting a difference between the expected Minerva success rate of approximately $78 \%$ and the lower confidence bound of the OPC of $66 \%$, required a minimum of 91 subjects. Assuming a dropout rate of $10 \%$ at 12 months, the required enrolled sample size was determined to be 102 subjects.

The Minerva system was studied at 7 clinical sites. Pooling of the data was justified by implementation of virtually identical protocols across all clinical sites and the methodology described by Meinert [22]. A Fisher-Freeman-Halton test of the rates of success was used to test whether the inves-

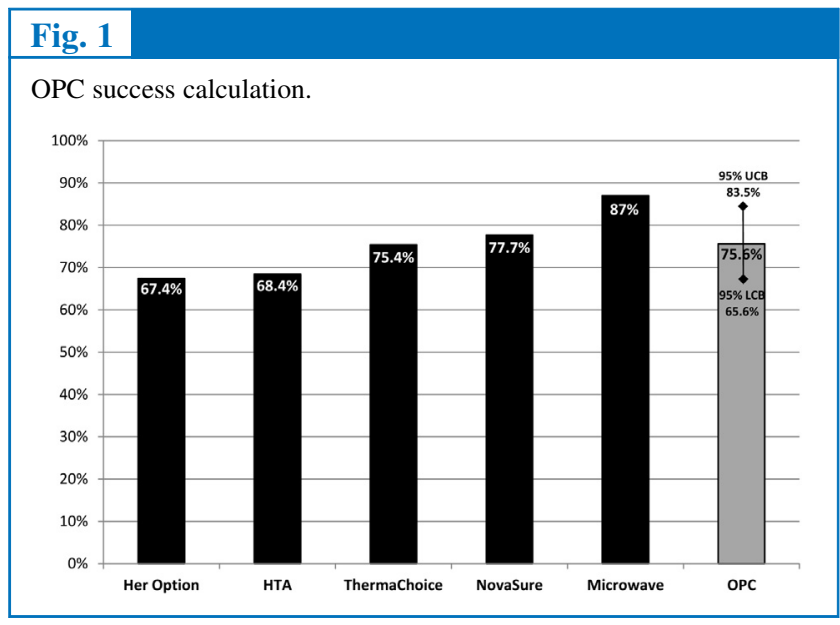

tigational sites differed in the primary endpoints with respect to primary effectiveness. The test for homogeneity of response was based on a 2-sided test at a 0.10 level of significance. Demographic and baseline characteristics were summarized descriptively by mean, standard deviation (SD), number evaluated, median, minimum and maximum for continuous variables and number evaluated, percentage, and $95 \%$ exact confidence limit on the percentage for categorical variables. Additional statistical methods were documented, and statistical soundness was ensured by the development of a formal statistical plan before study initiation. This plan outlined strict definitions and rules regarding data analysis related to subject accountability, missing data procedures, and safety, as well as effectiveness.

Statistical analysis was conducted by an independent contractor (Willes Consulting Group, Encinitas, CA) using SAS version 9.2 (SAS Institute, Cary, NC).

\section{Minerva Endometrial Ablation System: Principles of Operation}

The Minerva endometrial ablation system (Fig. 2) is designed to treat abnormal uterine bleeding due to benign

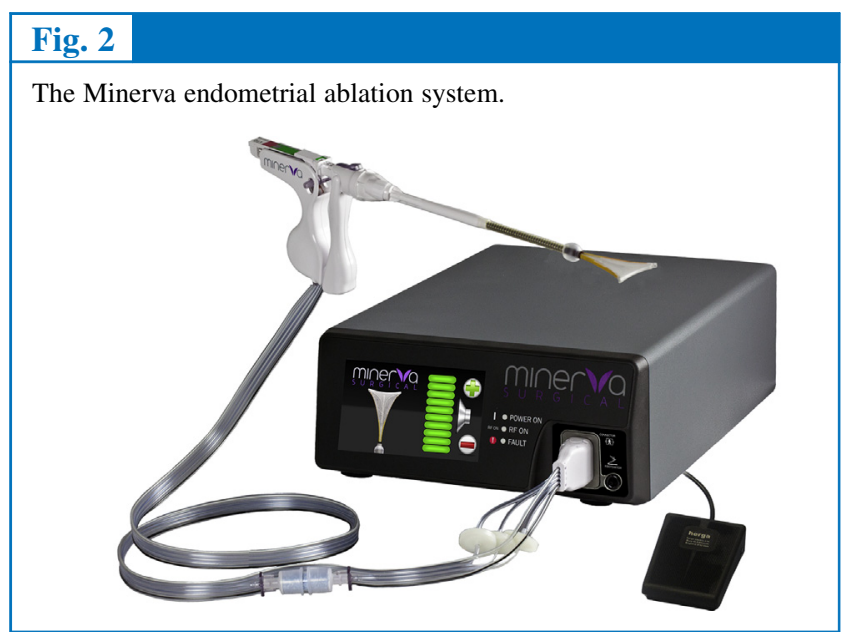




\section{Fig. 3 \\ The sealed silicone membrane covering the plasma formation array of the Minerva endometrial ablation system.}

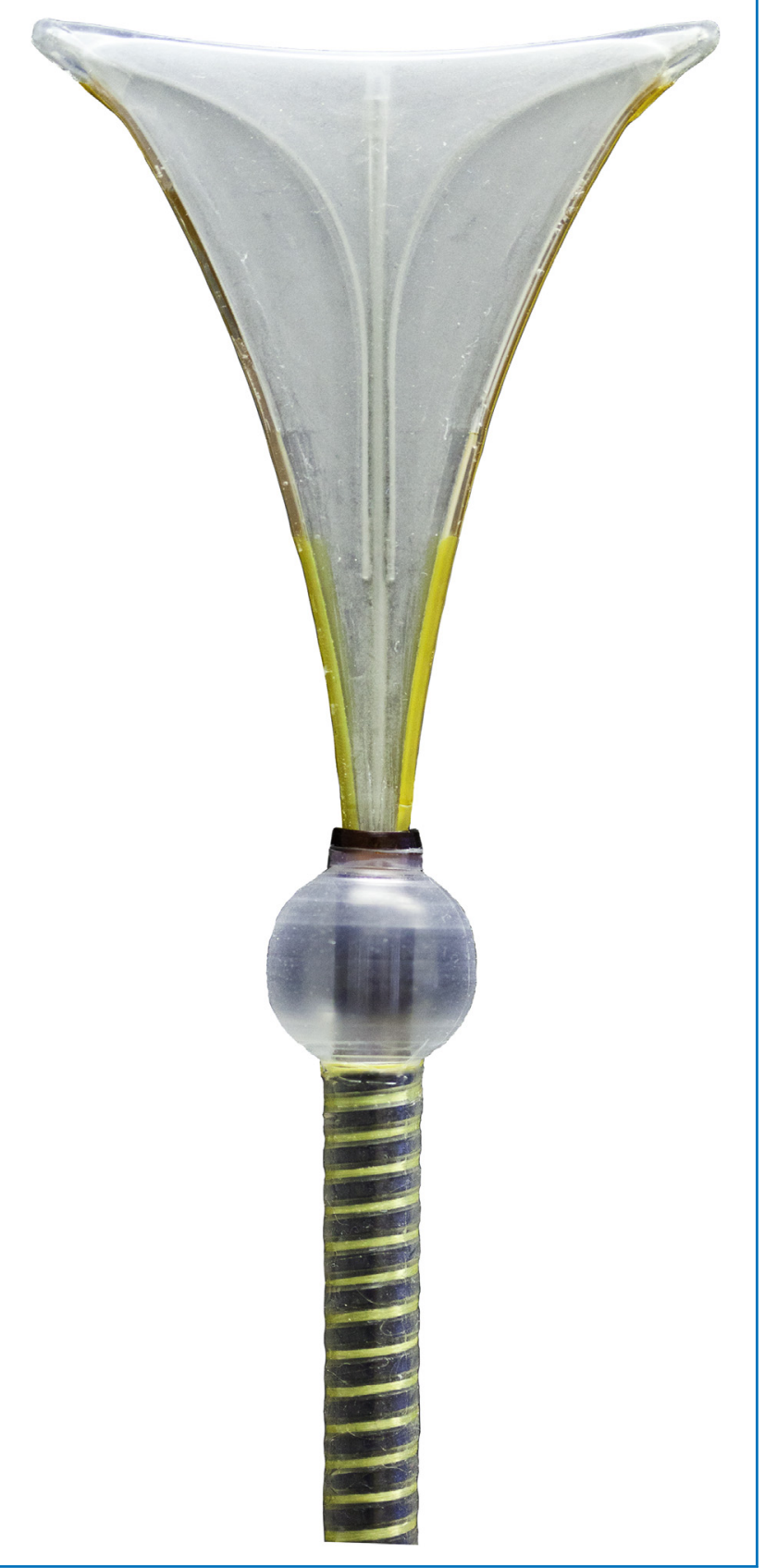

causes in premenopausal women for whom childbearing is complete. It consists of the Minerva surgical RF controller and the disposable endometrial ablation handpiece.

The Minerva endometrial ablation system uses bipolar RF electrical current at a frequency of $480 \mathrm{kHz}$ to ionize argon (Ar) gas, which is fully contained and circulating within a sealed silicone membrane covering the plasma formation array (PFA) (Fig. 3). This PFA is inserted and deployed in the uterine cavity. When the system is energized, the low-pressure $(\sim 25 \mathrm{mmHg})$ Ar gas that is circulating within the array membrane is ionized, transforming it into plasma. Moving at the speed of light, these ionized particles transform their kinetic energy into thermal energy on colliding with the silicone membrane. In this fashion, the Ar plasma heats the silicone membrane, and thermal energy is conducted into the adjacent endometrium.

During the ablation cycle, the Minerva system does not proactively evacuate the liquid contents from the uterine cavity. These liquids remain in the uterine cavity, are heated by the membrane, and are used to ablate the endometrial tissue that is not in direct contact with the membrane. This is especially helpful when the cavity is distorted by small intracavitary or intramural pathology or when the uterine cavity lacks axial symmetry. This "triple" mechanism of action combines direct membrane-to-tissue heat transfer with the ablative effect of the retained heated liquids, along with the tissue penetrating bipolar RF current in an impedancemediated resistive heating environment, creating a uniform and reproducible ablation of the endometrium.

\section{Minerva RF Controller}

The Minerva surgical RF controller is a RF power unit that generates, monitors, and manages energy delivery to the device. The power delivered to the disposable handpiece by the Minerva surgical RF controller varies according to electrophysical changes in the impedance characteristics of the endometrial tissue during the ablation process. As the ablation progresses and tissue impedance rises, the initial power level of approximately $40 \mathrm{~W}$ gradually drops correspondingly, reaching subtherapeutic levels at the end of the procedure. Whereas the total amount of energy delivered (power $[\mathrm{W}] \times$ time [seconds]) will vary from patient to patient, the total ablation time is a constant 2 minutes. In addition, the RF controller performs a uterine integrity test (UIT) designed to identify possible defects of the uterine wall and/ or the array membrane by introducing and monitoring the change (decay) in the flow rate of $\mathrm{CO}_{2}$ gas. The RF controller graphic user interface guides the user through each step of the procedure.

\section{Minerva Disposable Device}

The wet silicone membrane ensures a very low friction environment, facilitating easy insertion and deployment. The UIT is a preventive safety feature to help assess the uterine cavity for possible uterine and/or array membrane defects. During the UIT, $\mathrm{CO}_{2}$ gas is instilled into the space between the PFA membrane and the endometrial surface. Simultaneously, vacuum/suction is drawn on the inside of the PFA, allowing $\mathrm{CO}_{2}$ to freely fill the space outside the membrane. In this fashion, both the integrity of the uterine wall and the membrane are assessed by measuring $\mathrm{CO}_{2}$ flow decay. If the $\mathrm{CO}_{2}$ flow rate remains high, the UIT will produce an alarm and halt the procedure. If the flow rate 
drops and remains below a prespecified level, the system will automatically initiate the ablation cycle.

The distal tip of the sheath is equipped with a cervical sealing balloon that, when inflated, seals the cervical canal and the uterine cavity. On successful completion of the UIT, the ablation cycle starts automatically, and plasma energy is then delivered. With completion of the 2-minute ablation cycle, the cervical balloon is deflated, the array is closed, and the device is removed from the uterine cavity.

\section{Results}

\section{Efficacy Results}

A total of 110 premenopausal subjects with menorrhagia were included at 7 clinical sites. Informed consent was provided by each subject. Five subjects did not meet the inclusion criteria and were excluded during preoperative screening. The Minerva procedure was subsequently attempted in 105 subjects and was successful in all but 1 patient, who had a uterine cavity width of $<2.5 \mathrm{~cm}$ (exclusion criterion) at the time of the operative visit and underwent rollerball ablation outside of the study protocol.

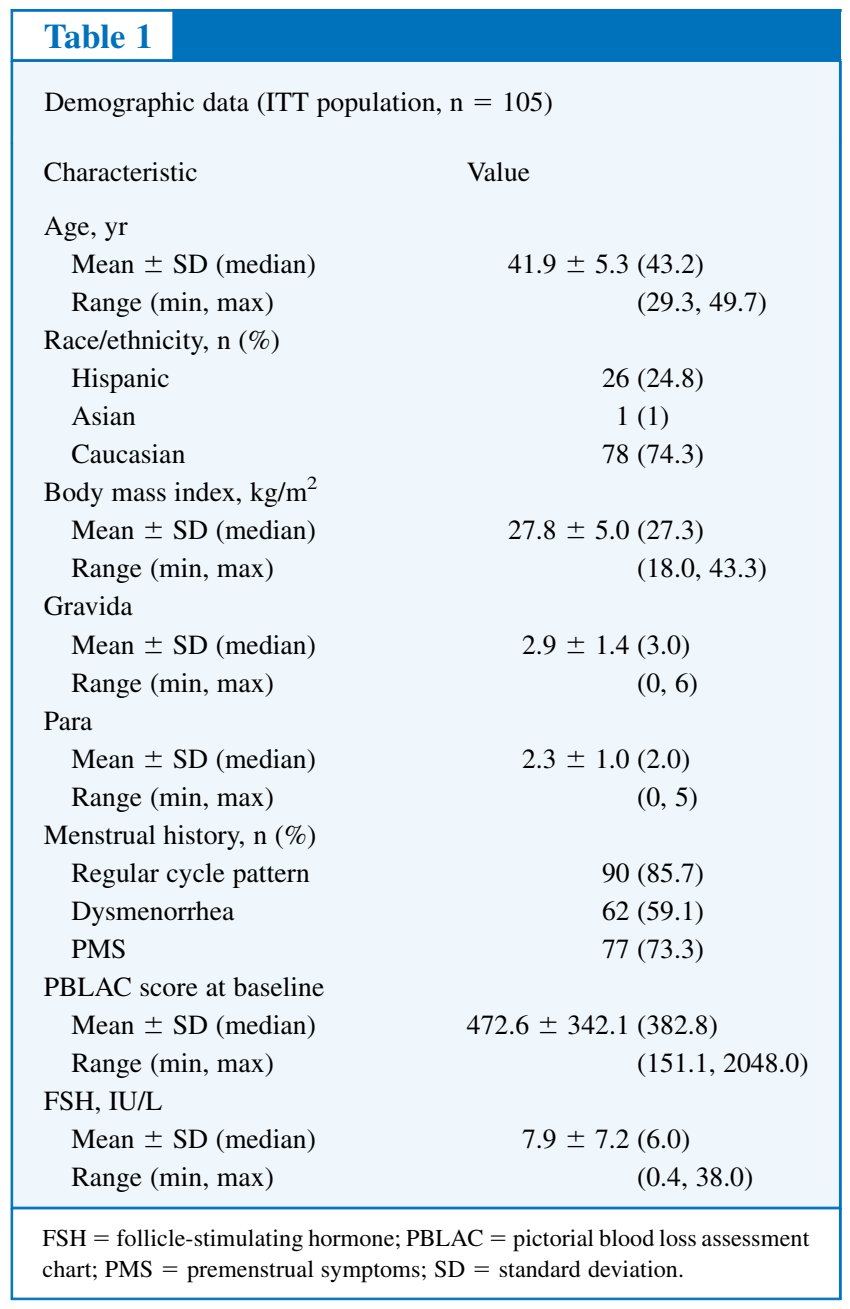

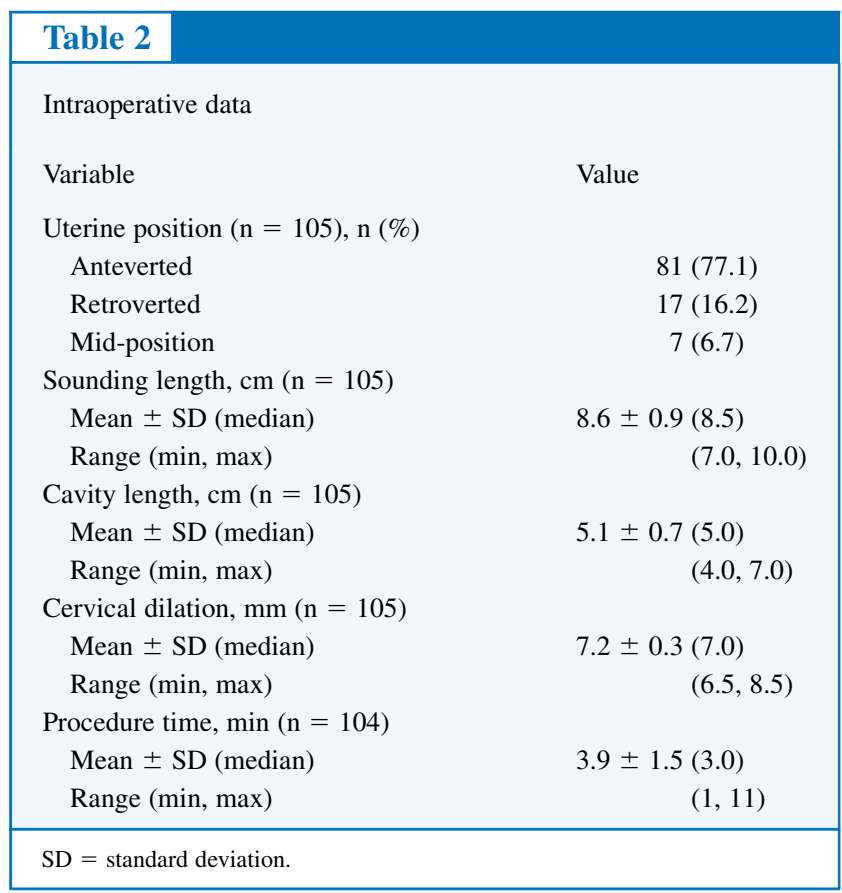

This subject was included in the intention-to-treat (ITT) group for calculating success and amenorrhea rates.

A total of 104 patients underwent the Minerva procedure, and all completed their 12-month follow-up visits. The homogeneity of the primary endpoint across investigational sites and countries was tested using the Fisher-FreemanHalton extension of Fisher's exact test. The assumption of homogeneity across both sites and countries was not rejected ( $\mathrm{p}=.14$ and 1.00, respectively). Because the hypothesis of homogeneity of response across sites and countries had been justified, and each investigational site conducted the study under a common protocol, we concluded that the primary endpoints may be pooled across study sites and countries for the effectiveness analyses.

Data on patient baseline demographics, gynecologic history, and other intraprocedural data are presented in Tables 1 and 2. The anesthesia regimens were ultimately left up to the discretion of each patient, investigator, and/or anesthesiologist while considering guidelines and anesthesia protocols adopted by each site. General anesthesia was used in $8.6 \%$ of the patients, paracervical block in $9.6 \%$, and intravenous sedation in $11.4 \%$. The balance received a combination of intravenous sedation, paracervical block, and other (unspecified) anesthesia regimens. The mean procedure time (interval from device insertion to device removal) was $3.9 \pm 1.5$ minutes (median, 3.0 minutes).

Treatment success was based on a reduction in diary score from PBLAC $>150$ pretreatment to PBLAC $\leq 75$ at the 1-year follow-up. The effectiveness (success) rate based on the ITT population at 1 year of follow-up was $96.2 \%$. The statistical hypothesis of this clinical study was supported, as detailed below. 


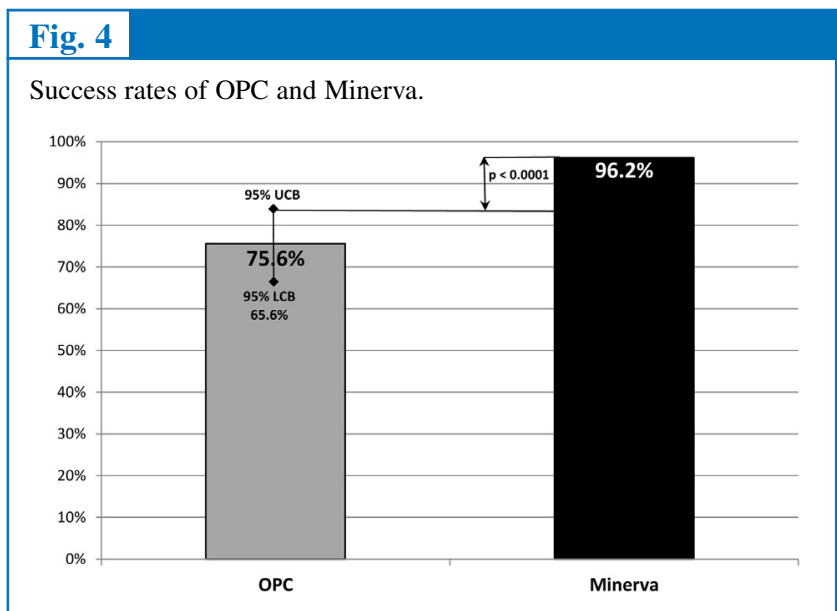

The purpose of the primary effectiveness analysis was to determine whether the Minerva success rate of $96.2 \%$ was statistically significantly equal to or lower than the OPC control's $95 \%$ lower confidence bound of $66 \%$. In a binomial test of the null hypothesis, based on the success rate of 96.2\% observed in the Minerva ITT population, the null hypothesis was rejected, and the 12-month follow-up success rate observed with the Minerva endometrial ablation system was demonstrated to be statistically significantly superior to the OPC's lower confidence bound of $66 \%$ ( $p<.0001)$. A secondary analysis performed using the same approach demonstrated that the $96.2 \%$ success rate of the Minerva procedure was statistically significantly superior to the OPC's 95\% upper confidence bound of $83.5 \%(\mathrm{p}<.0001)$ (Fig. 4).

The Minerva success rate was stratified and analyzed as a function of age at the time of the Minerva procedure. Patients were divided into 2 groups: age $\leq 40$ years and age $>41$ years. The treatment success rate of $97.4 \%$ (37 of 38 ) in 25 - to 40-year-olds was not statistically different from the $95.5 \%$ (64 of 67 ) reported in the 41 - to 50-year-olds ( $\mathrm{p}=$ 1.00, 2-sided Fisher's exact test).

Endometrial pretreatment (medical/surgical) was not used in this study, and the procedure was not timed to any particular menstrual cycle day. The procedure was performed even if the patient was actively bleeding at the time of the operative visit. As such, we conducted a separate stratification and comparison of effectiveness of the Minerva endometrial ablation system as a function of menstrual cycle phase at the time of the procedure. Only patients reporting a regular menstrual cycle $(n=90)$ were included in this analysis. Patients who underwent the Minerva procedure during the proliferative phase $(n=45)$ reported success rates similar to those who did so during the secretory phase (n $=45)(97.8 \%$ and $95.6 \%$, respectively; $p=1.00$, Fisher's exact test). In addition, success rates were not statistically different between subjects with irregular cycles $(\mathrm{n}=15)$ and those with regular cycles $(n=90)(93.3 \%$ vs $96.7 \% ; \mathrm{p}$ $=.47$, Fisher's exact test).

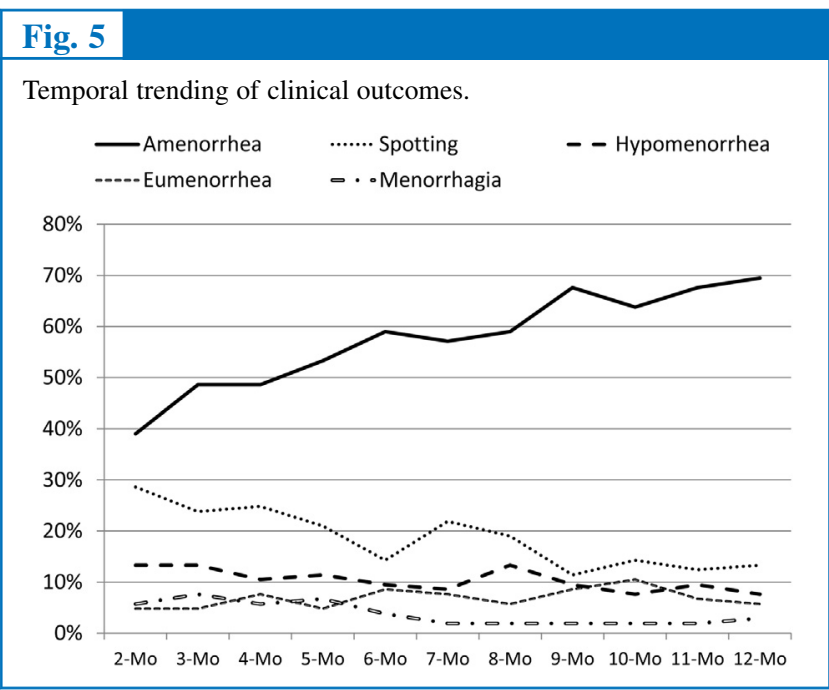

The secondary effectiveness objective was the rate of amenorrhea, defined as a PBLAC of 0 , at the at 12-month follow-up. Calculated for the ITT population of 105 subjects, this rate was $69.5 \%$.

During the 12-month follow-up period, no subjects required a hysterectomy or any additional medical and/or surgical interventions to control uterine bleeding. No patients were lost to follow-up during the study.

Bleeding diaries were collected monthly starting at month 2 and continuing through month 12. To assess bleeding pattern trends, PBLAC scores were categorized as amenorrhea, PBLAC 0; spotting: PBLAC 0.1 to 10; hypomenorrhea, PBLAC 10.1 to 30; eumenorrhea, PBLAC 30.1 to 75 ; or menorrhagia, PBLAC $>75$. Figure 5 shows bleeding trends over the course of the study. Listing absolute PBLAC scores by category demonstrates a trend toward an increasing rate of amenorrhea along with a decreasing rate of spotting. In other words, those with spotting tend to become amenorrheic, whereas those with hypomenorrhea,

\begin{tabular}{|lc|}
\hline Table 3 & \\
\hline Adverse events & \\
& \\
Adverse event & $\mathrm{n}(\%)$ \\
Anesthesia-related & $23(21.9)$ \\
Infection, vaginal & $1(1)$ \\
Bleeding & $7(6.7)$ \\
Endometritis & $1(1)$ \\
Pelvic cramping (nonspecific) & $71(67.6)$ \\
Pelvic cramping (dyspareunia) & $1(1)$ \\
Abdominal pain & $3(2.9)$ \\
Nausea more than 24 h posttreatment & $2(1.9)$ \\
Vaginal discharge & $14(13.3)$ \\
Urinary tract infection & $5(4.8)$ \\
Pelvic inflammatory disease & $1(1)$ \\
Fever $\left(>37^{\circ} \mathrm{C}\right)$ more than $24 \mathrm{~h}$ posttreatment & $2(1.9)$ \\
Other & $33(31.4)$ \\
Total & $105(100)$ \\
\hline
\end{tabular}


eumenorrhea, or menorrhagia are not likely to experience a significant change in bleeding pattern.

At study entry, subjects were asked whether they experienced premenstrual symptoms (PMS) and/or dysmenorrhea. For those with symptoms, $80.8 \%$ and $54.8 \%$ experienced a reduction in PMS and dysmenorrhea, respectively, at 12 months.

\section{Safety Results}

Safety of the Minerva endometrial ablation system was evaluated by recording all adverse events. Investigators conservatively recorded all signs and symptoms typically experienced following an endometrial ablation procedure (i.e., vaginal discharge, pelvic pain, abdominal cramping, vaginal bleeding, nausea, and vomiting) as adverse events (Table 3).

No serious or unanticipated device-related adverse events were recorded during the course of the study. The sole serious adverse event was not related to the study device or procedure. That patient required a preplanned artificial heart valve implant at approximately 8.5 months posttreatment. In addition, a 43-year-old patient conceived at 11 months after the Minerva procedure and subsequently underwent an uneventful dilation and curretage for a missed abortion. This event was adjudicated by the CEC/DMC as unrelated to the study device.

\section{QoL Results}

A menstrual impact questionnaire was administered at baseline and again at the 3-month, 6-month, and 12-month follow-up visits. Scores were assigned to each response, and a paired $t$-test was applied to each question listed to evaluate the change between baseline and the 12-month visit. For all questions, there was a statistically significant difference in the subjects' responses at 12 months compared with baseline (Table 4).

At the 12-month assessment of patient satisfaction with the Minerva procedure, $97.6 \%$ reported being "satisfied" or "very satisfied." Moreover, $98.8 \%$ stated that they would recommend the procedure to a friend or a relative, with only $1.2 \%$ reporting "not sure."

\section{Discussion}

Our methodology used a wide range of complementary study controls, including regulatory compliance; use of a CEC, a DSMB, and a statistical plan; and validation of the sanitary products for calculation of PBLAC score. Although complex and logistically challenging, this approach assured soundness of the study, generated the highest-quality data, and minimized bias during data interpretation. Compared with dropout rates as high as $25 \%$ in similar clinical trials, patient follow-up and compliance during this study were exemplary, with $100 \%$ completion of the 12-month visit. Such compliance may be attributable, among other factors, to the geographic location of the clinical sites, where subject population is in general less prone to migration.

The OPC approach for comparing the primary endpoint of a study (success) is widely used in both pharmacologic and medical device research. The present Minerva study is the first investigational study of endometrial ablation to use the OPC model in preparation for FDA approval of the device. The primary strength of this OPC study is its ability to compare the success rates of the Minerva system and the OPC composed of all 5 FDA-approved endometrial ablation systems, using data from their respective FDA pivotal RCTs. On the other hand, a potential weakness of

\begin{tabular}{|c|c|c|c|}
\hline \multicolumn{4}{|l|}{ Responses to the QoL questionnaire } \\
\hline Patients responding "sometimes," "frequently," or "always" to & Baseline, \% & 12-mo follow-up, $\%$ & $\mathrm{p}$ value \\
\hline Feel like yourself? & 78.5 & 96.3 & $<.0001$ \\
\hline Lack of desire or interest in sexual activities (if sexually active)? & 65.4 & 30.1 & $<.0001$ \\
\hline Feel you accomplished less than desired due to menses? & 78.16 & 9.63 & $<.0001$ \\
\hline Lack confidence in yourself due to menses? & 59.77 & 8.43 & $<.0001$ \\
\hline Have decreased pleasure or arousal during sexual activity (if sexually active)? & 51.2 & 13.2 & $<.0001$ \\
\hline Have difficulty performing work or other activities due to menses? & 78.17 & 9.63 & $<.0001$ \\
\hline Have difficulty concentrating? & 73.8 & 16.9 & $<.0001$ \\
\hline Have less energy or a total lack of energy due to menses? & 96.55 & 21.69 & $<.0001$ \\
\hline Feel you lacked control over your life? & 59.5 & 9.6 & $<.0001$ \\
\hline Spend less time at work or other activities outside your home due to menses? & 77.01 & 7.22 & $<.0001$ \\
\hline Feel tired or worn out? & 95.3 & 33.7 & $<.0001$ \\
\hline Have sexual relations less frequently than you desired due to menses (if sexually active)? & 66.71 & 13.2 & $<.0001$ \\
\hline Feel physically attractive? & 58.3 & 80.8 & $<.0001$ \\
\hline Feel anxious due to menses? & 74.72 & 12.04 & $<.0001$ \\
\hline Feel irritable? & 88.1 & 46.9 & $<.0001$ \\
\hline Miss athletic activities due to menses? & 77.02 & 6.02 & $<.0001$ \\
\hline Unable to work outside the home due to menses? & 57.46 & 7.22 & $<.0001$ \\
\hline
\end{tabular}


the study may be related to the fact that it lacked randomization, known to effectively address such important study elements like "washout" of population bias and blinding/ masking, which are better addressed using a RCT study model. This deficiency is currently being addressed through an ongoing RCT with rollerball ablation serving as the control.

The Minerva success rate of $96.2 \%$ compares favorably to the success rate of the OPC control. The statistically significant $(\mathrm{p}<.0001)$ superiority of the Minerva system over the OPC's 95\% upper confidence bound of $83.5 \%$ indicates that the Minerva system design is a potential improvement on the technical limits of comparative systems.

The reported amenorrhea rate of $69.5 \%$ for the Minerva system may be attributable to the system's use of the triple mechanism of action approach in endometrial tissue ablation. Simultaneous use of bipolar RF ablation, direct thermal energy transfer from membrane to tissue, and thermal energy transfer from the heated fluids present in the uterine cavity for the purpose of filling the potential gaps between the array and endometrium appears to provide a very interesting procedural solution. Theoretically, then, this device design allows for a more adequate ablation independent of uterine cavity geometry or adequacy of device deployment. Previous research has demonstrated that achieving amenorrhea is highly desirable and results in very high patient satisfaction rates [23], whereas failure to achieve amenorrhea commonly leads to hysterectomy [24]. Given that all patients were able to avoid hysterectomy at the 12-month follow-up, the $69.5 \%$ rate of amenorrhea coupled with the $96.2 \%$ success rate may serve to reduce the need for extirpative therapy in this population. These promising results require corroboration using longer follow-up to more fully evaluate, understand, and ultimately validate the role of the Minerva procedure.

Comparatively, the procedure time with the Minerva system was very short. On average, the total time from device insertion to device removal was 3.9 minutes. The degree of cervical dilation was similar to or less than that seen with the other devices. The short procedure time, small diameter, ease of device deployment, and low power level may allow for use of the Minerva system in the office setting. Intraoperative discomfort was not measured in this study and may be an area of interest for future research.

During the last decade, the impact of surgical treatment on overall QoL has been a key validator for clinical value. Research trends observed in Europe, Australia, and other countries indicate that QoL is even more important than other predefined and absolute values for study success (e.g., bleeding). In this study, on a validated QoL questionnaire administered 12 months after the procedure, subjects reported a statistically significant improvement in every category, including $97.6 \%$ who were satisfied with the outcome and $98.8 \%$ who would recommend the procedure to a friend. Along with reducing menstrual bleeding, the Minerva procedure reduced PMS by $80.8 \%$ and dysmenor- rhea by $54.8 \%$. The exact mechanism of PMS reduction after endometrial ablation is not understood and has been reported in other endometrial ablation research. Investigators theorize that this effect may result from reduced anxiety in anticipation of an excessive period after the condition has been successfully treated. Decreased dysmenorrhea, another known outcome of endometrial ablation, may be attributed to the possible effect of ablation on superficial adenomyosis, with endometrial glands located within the zone of ablation front effectively destroyed. It is critical to understand and appreciate that this type of outcome should not be promised to the patient during counseling, considering that endometrial ablation (of any kind) is not a solution in the management of adenomyosis.

The Minerva device was highly rated by the investigators with respect to ease of use and overall user satisfaction. The device was easy to insert and deploy, and required minimal to no seating. Use of the cervical sealing balloon for cervical canal occlusion during the UIT resulted in $100 \%$ adequate occlusion on the first attempt. The overall performance of the device was rated "excellent" or "good" in 98.1\% (102 of 104) of the treated cases. In all attempted cases, the investigators responded that they "would recommend use of the device."

\section{Conclusion}

This multicenter study demonstrates that at 12-month follow-up, the Minerva endometrial ablation system and procedure are safe and effective while producing results that were statistically significant superior compared with the OPC control. Moreover, the Minerva procedure produces high amenorrhea and patient satisfaction rates, is fast, easy to use, does not require general anesthesia and should be considered a minimally invasive treatment method of choice such that hysterectomy is ultimately avoided.

\section{References}

1. Goldrath MH, Fuller TA, Segal S. Laser photovaporization of endometrium for the treatment of menorrhagia. Am J Obstet Gynecol. 1981;140:14-19.

2. DeCherney AH, Diamond MP, Lavy G, Polan ML. Endometrial ablation for intractable uterine bleeding: hysteroscopic resection. Obstet Gynecol. 1987;70:668-670.

3. Vancaillie TG. Electrocoagulation of the endometrium with the ballend resectoscope. Obstet Gynecol. 1989;74:425-427.

4. Cooper JM, Brady RM. Late complications of operative hysteroscopy. Obstet Gynecol Clin North Am. 2000;27:367-374.

5. Lethaby A, Penninx J, Hickey M, Garry R, Marjoribanks J. Endometrial resection and ablation techniques for heavy menstrual bleeding. Cochrane Database Syst Rev. 2013;CD001501.

6. Meyer WR, Walsh BW, Grainger DA, Peacock LM, Loffer FD, Steege JF. Thermal balloon and rollerball ablation to treat menorrhagia: a multicenter comparison. Obstet Gynecol. 1998;92:98-103.

7. Corson SL. A multicenter evaluation of endometrial ablation by the Hydro ThermAblator and rollerball for treatment of menorrhagia. J Am Assoc Gynecol Laparosc. 2001;8:359-367.

8. Cooper J, Gimpelson R, Laberge P, et al. A randomized, multicenter trial of safety and efficacy of the NovaSure system in the treatment of menorrhagia. J Am Assoc Gynecol Laparosc. 2002;9:418-428. 
9. Duleba AJ, Heppard MC, Soderstrom RM, Townsend DE. A randomized study comparing endometrial cryoablation and rollerball electroablation for treatment of dysfunctional uterine bleeding. J Am Assoc Gynecol Laparosc. 2003;10:17-26.

10. Cooper JM, Anderson TL, Fortin CA, Jack SA, Plentl MB. Microwave endometrial ablation vs rollerball electroablation for menorrhagia: a multicenter randomized trial. J Am Assoc Gynecol Laparosc. 2004; 11:394-403.

11. Department of Health and Human Services, Food and Drug Administration. Summary of safety and effectiveness data: ThermaChoice uterine balloon therapy (UBT) system. Available at: http://www.accessdata. fda.gov/cdrh_docs/pdf/P970021.pdf. Accessed January 30, 2015.

12. Department of Health and Human Services, Food and Drug Administration. Summary of safety and effectiveness data: Her Option uterine cryoblation therapy system. Available at: http://www.accessdata.fda.gov/ cdrh_docs/pdf/P000032b.pdf. Accessed January 30, 2015.

13. Department of Health and Human Services, Food and Drug Administration. Summary of safety and effectiveness data: Hydro ThermAblator endometrial ablation system. Available at: http://www.accessdata.fda. gov/cdrh_docs/pdf/P000040b.pdf. Accessed January 30, 2015.

14. Department of Health and Human Services, Food and Drug Administration. Summary of safety and effectiveness data: NovaSure impedancecontrolled endometrial ablation system. Available at: http://www. accessdata.fda.gov/cdrh_docs/pdf/P010013b.pdf. Accessed January 30, 2015.

15. Department of Health and Human Services, Food and Drug Administration. Summary of safety and effectiveness data: microwave endometrial ablation system. Available at: http://www.accessdata.fda.gov/cdrh_ docs/pdf2/P020031b.pdf. Accessed January 30, 2015.

16. Laberge P, Leyland N, Murji A, Fortin C, Martyn P, Vilos G. Endometrial ablation in the management of abnormal uterine bleeding. J Obstet Gynecol Can. 2015;37:362-367.

17. National Women's Health Information Center. Hysterectomy. Available at: https://nwhn.org/hysterectomy. Accessed January 30, 2015.

18. Longinotti MK, Jacobson GF, Hung YY, Learman LA. Probability of hysterectomy after endometrial ablation. Obstet Gynecol. 2008;112: $1214-1220$

19. Thomassee MS, Curlin H, Yunker A, Anderson TL. Predicting pelvic pain after endometrial ablation: which preoperative patient characteristics are associated? J Minim Invasive Gynecol. 2013;20:642-647.

20. Wishall KM, Price J, Pereira N, Butts SM, Della Badia CR. Postablation risk factors for pain and subsequent hysterectomy. Obstet Gynecol. 2014;124:904-910.

21. Higham JM, O'Brien PM, Shaw RW. Assessment of menstrual blood loss using a pictorial chart. Br J Obstet Gynaecol. 1990;97:734-739.

22. Meinert CL. Clinical Trials: Design, Conduct, and Analysis. New York: Oxford University Press; 1986.

23. Weber AM, Munro MG. Endometrial ablation versus hysterectomy: STOP-DUB. Medscape Womens Health. 1998;3:3.

24. Aberdeen Endometrial Ablation Trials Group. A randomised trial of endometrial ablation versus hysterectomy for the treatment of dysfunctional uterine bleeding: outcome at four years. Br J Obstet Gynaecol. 1999;106:360-366. 